\title{
Survival from Septic Shock Secondary to Disseminated Group A Streptococcal Infection after Central Extracorporeal Membrane Oxygenation
}
A. Asfari ${ }^{1}$
M. Ahmed ${ }^{2}$
L. R. Edwards ${ }^{1}$
K. Irby ${ }^{1}$
A. Agarwal ${ }^{3}$
S. Pasala ${ }^{1}$
P. Prodhan ${ }^{1,4}$
B. Frazier $^{5}$
R. C. Sanders Jr. ${ }^{1}$

1 Section of Critical Care, Department of Pediatrics, Arkansas Children's Hospital, University of Arkansas for Medical Sciences, Little Rock, Arkansas, United States

2 Section of Critical Care, Department of Pediatrics, Texas Children's Hospital, Houston, Texas, United States

${ }^{3}$ Section of Pulmonology, Department of Pediatrics, Arkansas Children's Hospital, University of Arkansas for Medical Sciences, Little Rock, Arkansas, United States

${ }^{4}$ Section of Cardiology, Department of Pediatrics, Arkansas Children's Hospital, University of Arkansas for Medical Sciences, Little Rock, Arkansas, United States

${ }^{5}$ Department of Extracorporeal Membrane Oxygenation, Arkansas Children's Hospital, Little Rock, Arkansas, United States

\begin{abstract}
Address for correspondence A. Asfari, MD, Congenital Heart Center at UF Health Shands Children's Hospital, PO Box 100297, 1600 SW Archer Road, Gainesville, FL 32610-0297, United States (e-mail: ahmed.asfari@ufl.edu).
\end{abstract}

J Child Sci 2017;7:e130-e135.

\section{Abstract \\ Keywords \\ - central extracorporeal membrane oxygenation \\ - extracorporeal carbon dioxide removal \\ - group A $\beta$-hemolytic Streptococcus \\ - intensive care unit \\ - outcomes \\ - pediatric \\ - septic shock}

Objective The objective of this study was to describe a case of severe life-threatening acute respiratory distress syndrome (ARDS) and septic shock in a child who responded to a prolonged extracorporeal membrane oxygenation (ECMO) support course utilizing different cannulation techniques depending on the physiological derangement until he recovered.

Design This is a case report.

Setting This study was done at the medical-surgical pediatric intensive care unit in an academic freestanding children's hospital.

Patient A previously healthy 4-year-old boy was presented with respiratory distress and fever. He was diagnosed with respiratory syncytial viral upper respiratory tract infection and group A $\beta$-hemolytic Streptococcus septic shock.

Interventions The patient was referred to peripheral ECMO for hemodynamic, ventilatory, and oxygenation support; conversion to central ECMO to augment blood flow; and transition to extracorporeal carbon dioxide removal before successful wean off extracorporeal support.

Measurements and Main Results Patient experienced severe pediatric ARDS and septic shock that were refractory to maximal medical therapy. Patient was able to be decannulated after 75 days of extracorporeal support. He was weaned completely off of mechanical ventilation and oxygen after 6 months. The only neurological deficit he exhibited was poor fine motor skills of his hands for which he continued to receive physical therapy. received

July 22, 2017

accepted after revision

September 8, 2017
DOI https://doi.org/

$10.1055 / \mathrm{s}-0037-1607312$.

ISSN 2474-5871.
Copyright $\odot 2017$ Georg Thieme Verlag License terms

KG Stuttgart · New York c) $(1) \$$ 
Conclusion Central ECMO may benefit children with pediatric ARDS and septic shock who require higher flows than what can be provided from peripheral ECMO. Extracorporeal membrane carbon dioxide removal may be an effective option in children who do not respond to mechanical ventilation alone.

\section{Introduction}

A previously healthy 4-year, 10 -month old boy (18 kg) was seen by his pediatrician for a fever to $101^{\circ} \mathrm{F}$ and an overnight "barky cough." He received an oral dose of dexamethasone and was discharged home. Later that afternoon, he developed increased respiratory distress and was evaluated in an outside hospital. He received a $20-\mathrm{mL} / \mathrm{kg}$ normal saline intravenous (IV) bolus, a racemic epinephrine nebulization, and IV dexamethasone. Due to concerns of impending respiratory failure, he was transferred to a higher level of care facility.

Upon arrival of the transport team, he was breathing at a rate of 60 breaths/min while receiving $15 \mathrm{~L} / \mathrm{min}$ of oxygen via a nonrebreathing facemask. He was restless, but warm and well perfused with strong pulses. His breathing increased during transport and he was placed on continuous positive airway pressure at $10 \mathrm{~cm} \mathrm{H}_{2} \mathrm{O}$. Despite maximal medical interventions, he required immediate intubation and mechanical ventilation due to hypoxemic respiratory failure when he arrived to the pediatric intensive care unit (PICU).

Later in the evening, he was found to have anisocoria and was given hypertonic ( $3 \%$ ) saline. An emergent head computed tomography scan (CT scan) was normal. Approximately 17 hours after admission, an arterial blood gas demonstrated a pH of 7.19, $\mathrm{PaCO}_{2}$ of $50 \mathrm{~mm} \mathrm{Hg}, \mathrm{PaO}_{2}$ of $85 \mathrm{~mm} \mathrm{Hg}$, and a base deficit of -9.1 . He was receiving conventional mechanical ventilation in the pressure-regulated volume control modality with a tidal volume of $6 \mathrm{~mL} / \mathrm{kg}$, positive end-expiratory pressure of $14 \mathrm{~cm} \mathrm{H}_{2} \mathrm{O}$, rate of 30 , and $\mathrm{FiO}_{2}$ of $70 \%$ while in the prone position. His peak inspiratory pressure (PIP) was $33 \mathrm{~cm} \mathrm{H} \mathrm{H}_{2} \mathrm{O}$. Due to hemodynamic instability, patient continued to receive large crystalloid and colloid volume resuscitation. His net fluid balance at this point was close to 2.7 positive. His chest X-ray revealed patchy peripheral and bilateral lower lobe opacities with left pleural effusion (-Fig. 1A). Given his worsening metabolic acidosis, increasing pulmonary edema, and elevated central venous pressure, an echocardiogram was ordered to assess his cardiac function. He was noted to have good systolic function but diastolic dysfunction. Milrinone was initiated at a continuous IV rate of $0.3 \mu \mathrm{g} / \mathrm{kg} / \mathrm{h}$. A complete blood count demonstrated a white blood cell count of 660 cells $/ \mathrm{mm}^{3}(64 \%$ lymphocytes, $24 \%$ segmented neutrophils, 188 absolute neutrophil count, and 422 absolute lymphocyte count). Polymerase chain reaction analysis for respiratory syncytial virus from a nasopharyngeal secretion sample was positive. His throat and blood cultures grew group A Streptococcus (GAS) bacteria approximately 19 hours after admission. He was treated empirically with cefotaxime and clindamycin. He also received IV immunoglobulin G (IVIG) and plasmapheresis.

The patient continued to require volume resuscitation and was treated with escalating doses of continuous vasopressor infusions including dopamine $20 \mu \mathrm{g} / \mathrm{kg} / \mathrm{min}$, epinephrine $0.2 \mu \mathrm{g} / \mathrm{kg} / \mathrm{min}$, norepinephrine $0.2 \mu \mathrm{g} / \mathrm{kg} / \mathrm{min}$, and milrinone $0.5 \mu \mathrm{g} / \mathrm{kg} / \mathrm{min}$. Vancomycin was added to his antibiotic regimen. A repeat echocardiogram revealed diminished left ventricular systolic function. Due to signs of inadequate oxygen delivery and hypoxemia, inhaled nitric oxide was introduced at $20 \mathrm{ppm}$. During this time, he developed a right-sided tension pneumothorax that was managed with a right-sided chest tube (-Fig. 1B, C). Despite these measures, his metabolic acidosis persisted and his lungs remained poorly compliant with PIPs ranging from 45 to $50 \mathrm{~cm} \mathrm{H}_{2} \mathrm{O}$. He went into cardiac arrest twice requiring cardiopulmonary resuscitation for approximately 2 and 15 minutes, respectively. His serum lactate levels continued to rise (up to $9 \mathrm{mmol} / \mathrm{L}$ ) despite maximal medical support, thus the decision was made to electively place him on peripheral venoarterial extracorporeal membrane oxygenation (VA ECMO). He was cannulated via his right internal jugular vein with a 22-French catheter and his right carotid artery with a 16-French catheter. Shortly after peripheral VA ECMO initiation, his heart went into asystole and his lung fields appeared to have near total opacification (-Fig. 2B). He developed anasarca and exhibited intermittent anisocoria. Over the following 22 hours, his venous return progressively worsened despite aggressive fluid administration and inotropic support. In addition, he became anuric and developed abdominal compartment syndrome, and at this point, his net fluid balance was more than $5 \mathrm{~L}$ positive.

Thus, his peripheral VA ECMO support was converted to open chest central VA ECMO support via a median sternotomy with the addition of a 24-French venous catheter to his right atrium. In addition, his left atrium was vented with a 13 -French catheter. The 16-French arterial catheter was left in the right carotid artery. His heart was observed to be markedly enlarged. Significant bilateral pleural effusions were drained directly via his sternotomy incision. Two chest tubes and a mediastinal drain were placed and the sternotomy was covered with a Silastic membrane. A Tenckhoff peritoneal catheter was also placed after a significant amount of ascites was surgically drained. After the addition of transthoracic cannulas, he had rapid and substantial improvement in his ECMO flows increasing from 67 to $88 \mathrm{~mL} / \mathrm{kg} / \mathrm{min}$ in peripheral ECMO to 150 to $200 \mathrm{~mL} / \mathrm{kg} / \mathrm{min}$ after central cannulation (patient's dry weight was $18 \mathrm{~kg}$ at admission). His lactic acidosis improved as well. Over the following week, his cardiac function improved. On day 8 of VA ECMO, his transthoracic cannulas were removed, and he was transitioned back to full support via his right neck vessels (peripheral VA ECMO). Patient received continuous renal replacement therapy while on central ECMO, his kidney function started to improve gradually, and he required slow continuous ultrafiltration for fluid removal intermittently through his course. 

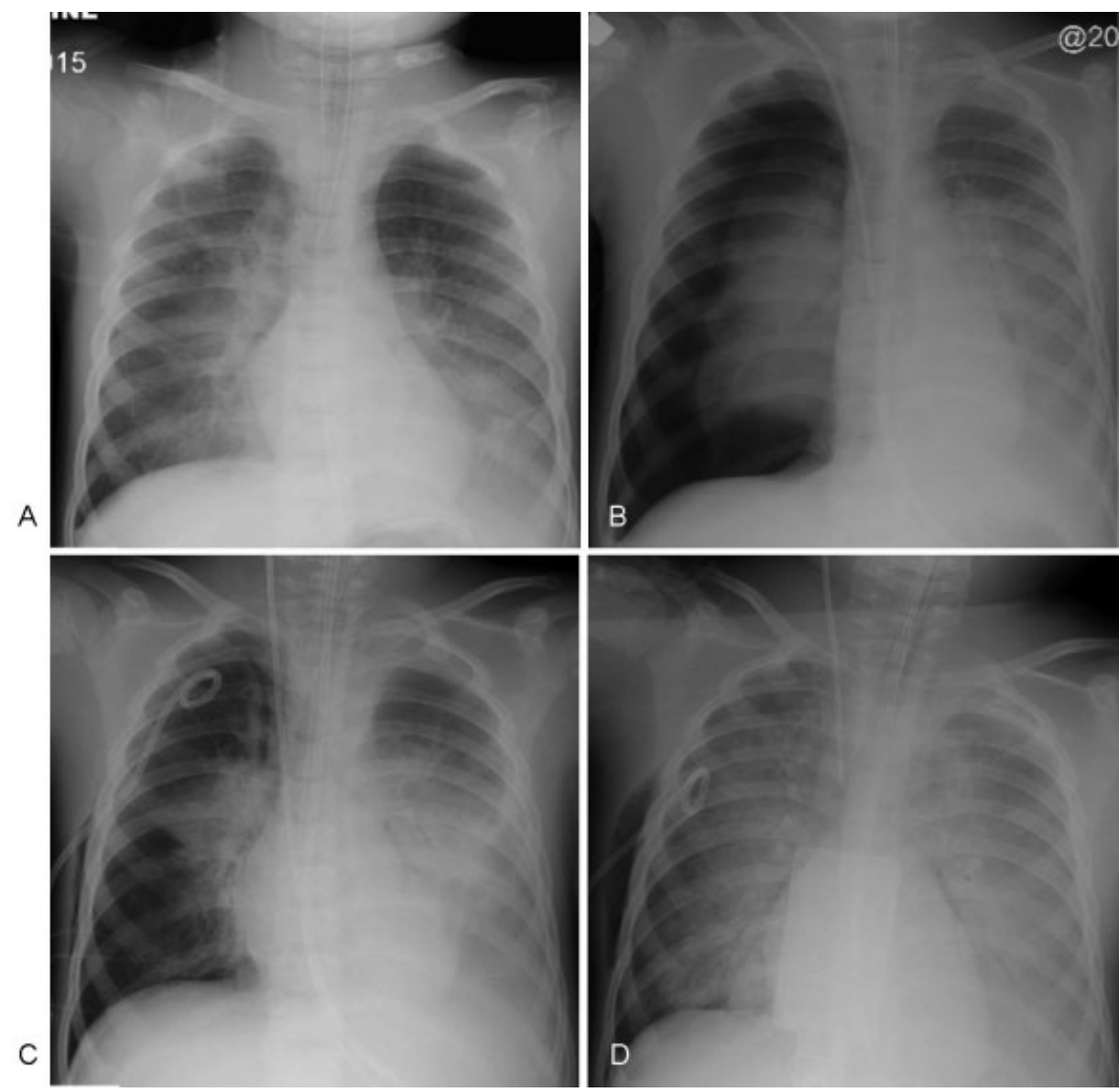

Fig. 1 (A) CXR on the day of admission. Patchy peripheral and lower lobe opacities (left greater than right). (B) CXR the next evening revealing a large right-sided pneumothorax with lung collapse along with probable mild to moderate left-sided pleural effusion. (C) CXR postright chest tube placement. (D) CXR postcardiac arrest a few hours later. Bilateral haziness consistent with pulmonary edema. Persistence of mild to moderate left-sided pleural effusion. CXR, chest X-ray.

Approximately 1 month into his VA ECMO support, he developed septic shock secondary to Hemophilus influenzae that was isolated from his blood, respiratory secretions, and pericardial fluid. He experienced significant bleeding from his mediastinum. Efforts were made to minimize his anticoagulation by maintaining antifactor Xa levels in the range of 0.3 to 0.4 while normalizing his prothrombin time and platelet levels (goal of $>100,000 / \mu \mathrm{L}$ ). His antibiotic regiment of cefepime and meropenem was simplified to ceftriaxone to target GAS and $H$. influenzae.

On day 37 of VA ECMO, mediastinal debridement was performed due to concerns that he had developed mediastinitis. At this time, he was found to have a bronchopleural fistula originating from his right lung. The area was packed with a kaolin impregnated sterile gauze (QuickClot). To limit the positive pressure reaching his right lung and the fistula to heal, the otolaryngology service performed bronchoscopy and placed his endotracheal tube in the left main bronchus. After 2 days, mediastinal re-exploration was performed and the kaolin impregnated sterile gauze was removed with no resulting air leak. After 7 days, the endotracheal tube was pulled back to its typical position above the carina. His mediastinum was gradually closed over the course of several weeks.

Multiple efforts to wean him off of VA ECMO support and transitioning to VV ECMO were unsuccessful because of severe respiratory acidosis even and the liable hemodynamic status. On day 59 of VA ECMO support, he was transitioned to pumpless extracorporeal $\mathrm{CO}_{2}$ removal $\left(\mathrm{ECCO}_{2} \mathrm{R}\right)$ by placing a 14-French cannula in both his left femoral artery and left femoral vein. His peripheral (right neck) ECMO cannulas were removed at that time. Over a few weeks, his $\mathrm{ECCO}_{2} \mathrm{R}$ support was gradually weaned. He was decannulated from $\mathrm{ECCO}_{2} \mathrm{R}$ on day 75 of total extracorporeal support.

While on ECMO, patient was on Rotaflow ECMO system. While he was on VA ECMO, flow range was 2 to $3.5 \mathrm{~L} / \mathrm{min}$, sweep varied greatly depending on age of oxygenator, his sweep range between 2.5 and $8 \mathrm{~L} / \mathrm{min}$ with higher sweep resulting in frequent oxygenator changes for clotting and poor gases. $\mathrm{FiO}_{2}$ range between 50 and $100 \%$ for majority of his run up until $\mathrm{ECCO}_{2} \mathrm{R}$.

While on $\mathrm{ECCO}_{2} \mathrm{R}$, patient was on Rotaflow ECMO system. He started on pediatric Quadrox oxygenator, but was changed to adult Quadrox within 12 hours to achieve higher flow rate. He remained on adult Quadrox for the remainder of his $\mathrm{ECCO}_{2} \mathrm{R}$. $\mathrm{ECCO}_{2} \mathrm{R}$ flow ranged between 0.4 and $0.5 \mathrm{~L} / \mathrm{min}$. Sweep rate ranged between 2 and $3 \mathrm{~L} / \mathrm{min}$ at $21 \% \mathrm{FiO}_{2}$.

Heparin was used for anticoagulation with activated clotting time (ACT) goal of 160 to 180 . Due to bleeding complications from chest, ACT range was lowered to 140 to 


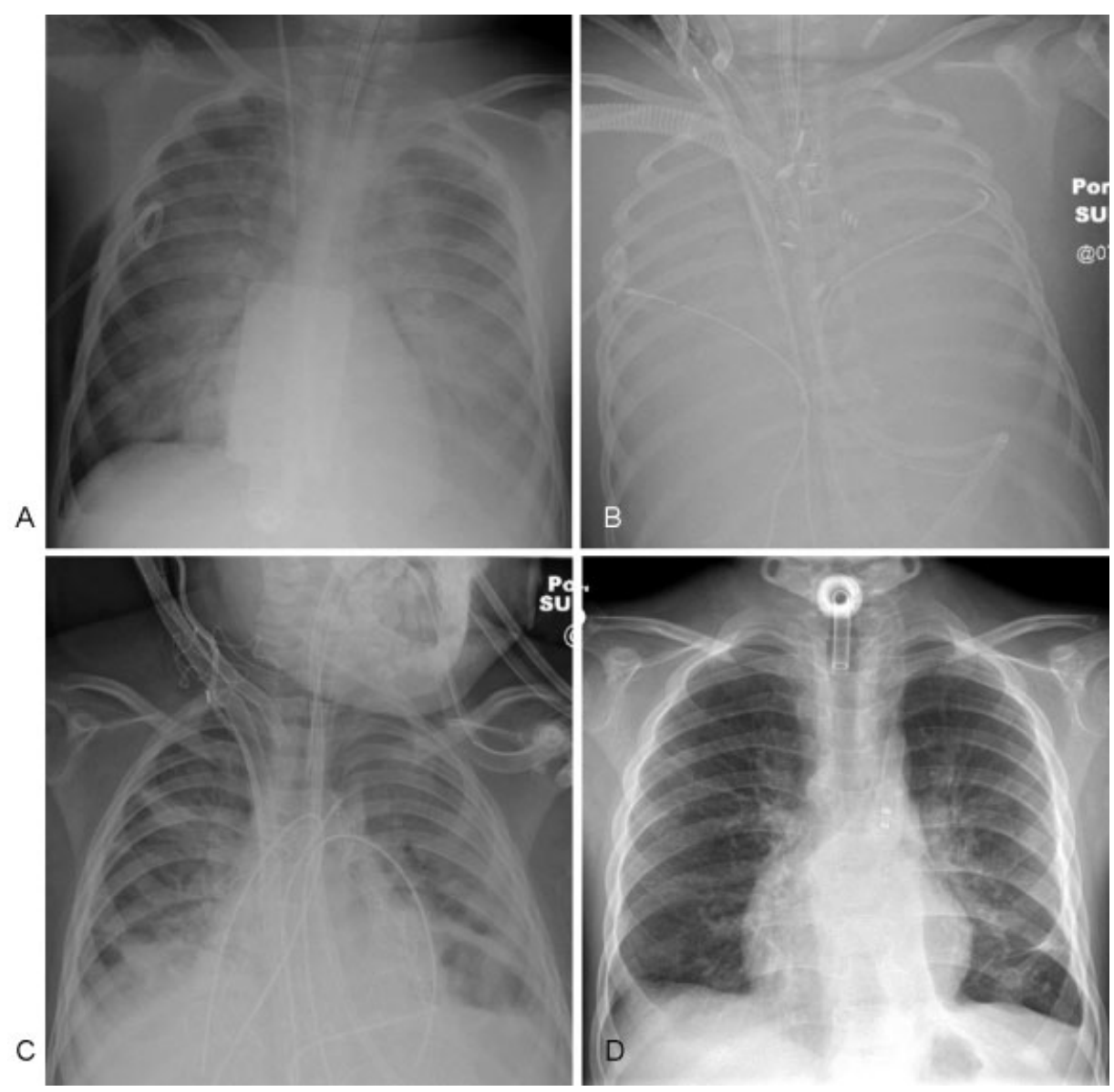

Fig. 2 (A) CXR after initial cardiac arrest. Increasing pulmonary edema. (B) CXR postECMO cannulation. Bilateral pulmonary edema. Increased right-sided emphysema. Decreased pneumothorax. Left-sided mild to moderate pleural effusion. (C) CXR approximately 6 weeks on ECMO. ECMO cannulas present. Bibasilar atelectasis with bilateral pleural effusions. (D) CXR 9.5 months after admission. Residual left lower lobe opacity with possible scarring noted in the right upper lobe and left lower lobe regions. Blunting of both costophrenic sulci with possible chronic small bilateral pleural effusions. CXR, chest X-ray; ECMO, extracorporeal membrane oxygenation.

160 for majority of treatment. Heparin infusion was often at variable doses between 40 and 50 units $/ \mathrm{kg} / \mathrm{h}$.

After 4.5 months of hospitalization, tracheostomy and gastrostomy tubes were placed. On day 167 of hospitalization, efforts to sprint him off of the ventilator to a tracheostomy collar began. The patient was discharged from the hospital requiring night time ventilation through the tracheostomy tube. Overall, he spent 229 days in the hospital, 152 days in the PICU and 77 days in the rehabilitation unit. At the time of his discharge, the only neurological deficit that he exhibited was poor fine motor skills in his hands for which he continued to receive physical therapy. He was discharged home on amlodipine for systemic hypertension, sildenafil for pulmonary hypertension, albuterol, and ipratropium.

Gradually, he was successfully weaned off of the ventilator and supplemental oxygen over a period of 6 months. He tolerated the capping of his tracheostomy tube throughout the day. He experienced shortness of breath while running, but he was able to engage in normal activity without any significant respiratory problems. Fourteen months after his original admission, spirometry revealed low vital capacity at $59 \%(0.84 \mathrm{~L})$ of predicted value for his age; nose clips and Microfoam tape around the tracheostomy were used to mini- mize air leak during testing. These observations were consistent with restrictive lung disease. A polysomnogram was performed with the tracheostomy tube capped and demonstrated normal oxygenation and ventilation.

A follow-up chest radiograph revealed minor residual atelectasis in the left lower lobe with small bilateral chronic pleural effusions ( - Fig. 2D). A chest CT scan demonstrated traction bronchiectasis, atelectasis, and consolidation of bilateral lower lung lobes greater on the left compared with the right (-Fig. 3). Serial echocardiograms did not show any evidence of pulmonary artery hypertension; therefore, sildenafil was successfully weaned off.

\section{Discussion}

Streptococcus pyogenes is a $\beta$-hemolytic bacterium that is also known as GAS. Invasive GAS accounts for 8,950 to 11,500 cases and 1,050 to 1,850 deaths each year in the United States. ${ }^{1}$ The rates of disease are highest among children who are younger than 1 year of age and persons older than 65 years of age. ${ }^{1}$ GAS is responsible for a spectrum of illnesses in children, ranging from pharyngitis to fatal invasive disease, ${ }^{2,3}$ and it is an emerging cause of severe sepsis in children. ${ }^{4}$ 

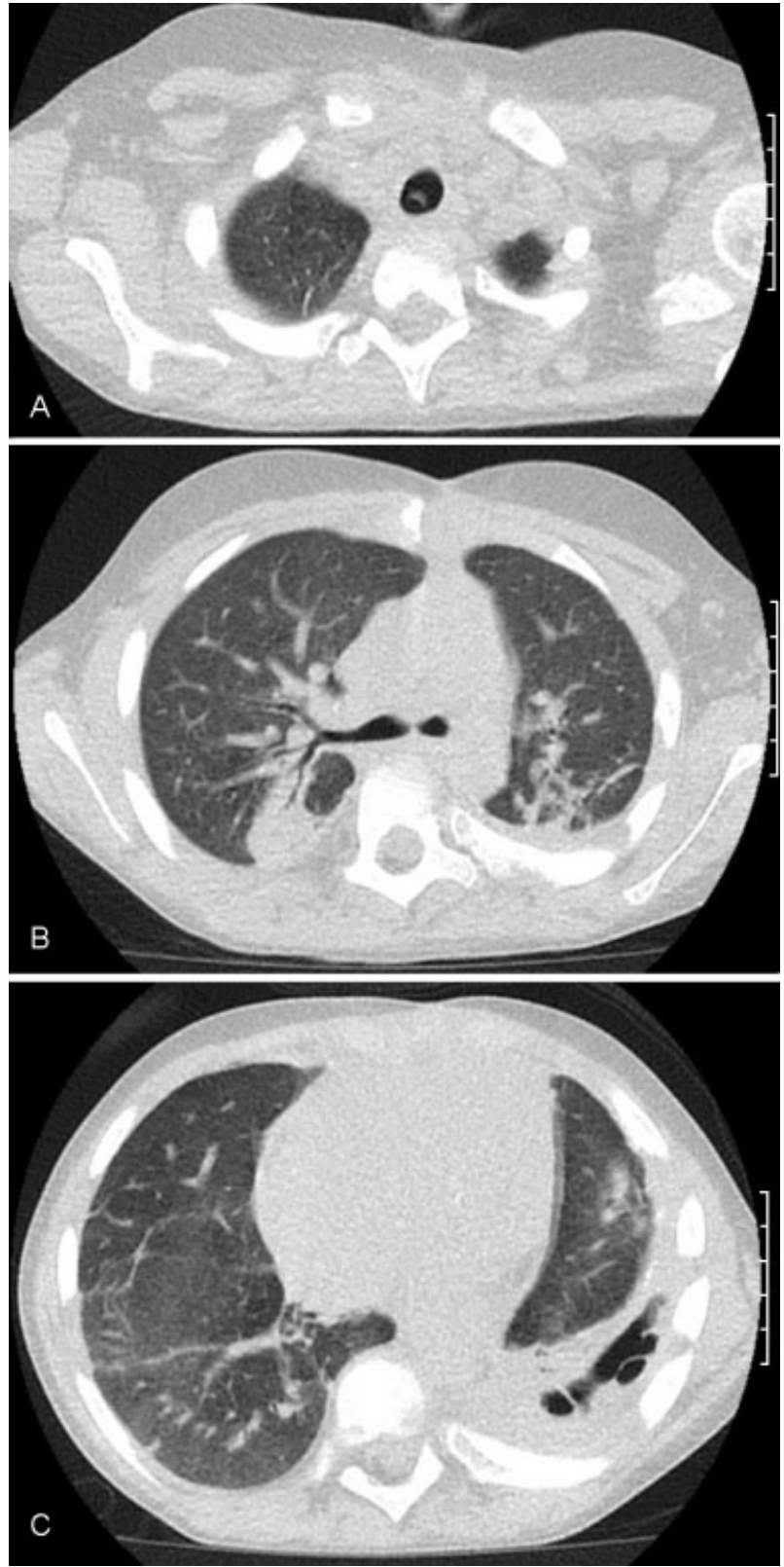

Fig. $3 \mathrm{CT}$ scan of the chest 6.5 months after admission. Interval improved aeration of the right lung and in the left upper lobe. Persistent atelectasis, fibrotic scarring of the left lower lobe resulted with increasing cystic areas, and bronchiectasis changes. Diffuse peribronchial thickening in bilateral peripheral region with extension of fibrotic changes persist. (A) Section of CT scan depicting lung apices above the carina. (B) Section of CT scan near divergence of the main stem bronchi. (C) Section of the CT scan just above the diaphragm. CT, computed tomography.

In one case series from Melbourne over a 3-year period, 12 cases of invasive group A streptococcal infection were admitted to the PICU. ${ }^{4}$ The most common clinical presentations were pneumonia, bacteremia with no septic focus, and septic arthritis. Fever and hypotension were common signs, but a rash was infrequent. The typical patient had multiorgan dysfunction including some combination of acute respiratory distress syndrome (ARDS), renal failure, liver dysfunction, and coagulopathy. ${ }^{4}$ Half of the patients required ECMO support ranging from 4 to 35 days though there was no mention of which type of ECMO support, peripheral or central, was needed. ${ }^{4}$

Initial management of invasive GAS infections is centered on early antimicrobial therapy, ensuring adequate cardiac output and supporting failing organs. ${ }^{2}$ A two-pronged parenteral antimicrobial therapy strategy is recommended utilizing a bactericidal agent ( $\beta$-lactamase-resistant antimicrobial) and a protein synthesis inhibiting agent (e.g., clindamycin) to diminish production of virulence factors. ${ }^{5}$ IVIG should be considered for those refractory to escalating hemodynamic support and meeting criteria for streptococcal toxic shock syndrome. ${ }^{5,6}$

The use of ECMO is part of the clinical practice parameters guidelines for hemodynamic support of pediatric and neonatal septic shock. ${ }^{7}$ The procedure is a form of cardiopulmonary bypass, modified for longer term support. The patient's venous blood is emptied from the body via a large bore cannula, and then, it is directed through a membrane oxygenator where carbon dioxide is removed and oxygen is added before being returned into the venous or arterial circulation. ${ }^{8}$ The typical insertion sites of the cannulas for peripheral ECMO include the large vessels of the neck and/or groin. In central ECMO, the venous cannula is placed in the inferior vena cava or right atrium (in a transthoracic approach), while the arterial cannula is inserted into the ascending aorta by way of a median sternotomy. In general, patients with isolated respiratory or cardiac failure are successfully supported using ECMO flows that can be achieved using peripheral ECMO. Physical properties of the length and diameter of the catheters and the size of the vascular bed being drained play a role in the limitation of ECMO flow. ${ }^{9}$ Our patient benefited from the higher flows achieved with central ECMO as well as decompressing the tamponade caused by extensive pleural edema and effusion from disease severity and fluid resuscitation.

We are certainly not the first institution to use central ECMO $^{10-13}$ for support of refractory septic shock, in either children ${ }^{11-14}$ or adults. ${ }^{15}$ MacLaren et al published a case series of children, many with overwhelming meningococcemia, who had good outcomes utilizing central ECMO support in the setting of septic shock by aiming to achieve "supernormal" ECMO flow to improve tissue bed perfusion, overcome vasomotor paralysis, and resolve lactic acidosis. ${ }^{14}$ While the successful use of central ECMO in septic shock has been reported, its use in children still seems to be merely a consideration rather than an expectation. At the time of this patient's presentation, we had extensive experience using peripheral ECMO for support of septic shock, but often with disappointing results due to the inability to achieve the desired supernormal ECMO flow. Our institution had extensive experience with transthoracic ECMO support for cardiac failure, often postoperatively. Given these experiences and the willingness of our cardiac surgical team to attempt to support this patient with central ECMO, making the imaginative leap to put into practice the lessons taught by our Australian colleagues was a relatively easy one. In the largest Australian series reported using central ECMO support for refractory septic shock, survival was statistically associated with maximal ECMO flow achieved with survivors able to reach ECMO flows of 158 versus $111 \mathrm{~mL} / \mathrm{kg} / \mathrm{min}$ for nonsurvivors. ${ }^{14}$ For our patient, ECMO flow increased from 67 to $88 \mathrm{~mL} / \mathrm{kg} / \mathrm{min}$ in peripheral ECMO to 150 to $200 \mathrm{~mL} / \mathrm{kg} / \mathrm{min}$ after central cannulation. It should be noted that these ECMO flows 
are approximately $50 \%$ higher than those typically recommended for venoarterial support of either primary respiratory failure or primary cardiac failure.

The use of $\mathrm{ECCO}_{2} \mathrm{R}$ to "bridge" our patient off VA ECMO support was a first for our institution, though use of $\mathrm{ECCO}_{2} \mathrm{R}$ for support of respiratory failure associated with severe hypercapnia is certainly not novel, particularly in adult critical care medicine. $\mathrm{ECCO}_{2} \mathrm{R}$, also discussed in the literature as "lowflow ECMO," may be performed in a venovenous or an arteriovenous manner, analogous to continuous renal replacement therapy. ${ }^{16}$ It allows use of ultraprotective ventilation strategies with very small tidal volumes and transalveolar pressures. ${ }^{16}$ Scattered case reports of the use of $\mathrm{ECCO}_{2} \mathrm{R}$ in pediatric ARDS (PARDS) have been in existence since the early $1990 s,{ }^{17}$ but given that the primary derangement in PARDS tends to be hypoxemia rather than hypercapnia, ${ }^{18-20}$ most pediatric respiratory ECMO support has traditionally used "high-flow" ECMO, either venoarterial or venovenous. ${ }^{8,10}$ Our patient's ARDS caused hypercapnia rather than hypoxemia off ECMO support, beside the fact that the $\mathrm{ECCO}_{2}$ requires less anticoagulation and are associated with less complication; we were encouraged to use it instead of VV ECMO as a bridge to wean our patient off of ECMO support.

\section{Conclusion}

Group A streptococcal infection can present with a wide range of clinical symptoms from fever and sore throat to severe lifethreatening sepsis. Our patient's symptoms evolved acutely and he remained hemodynamically unstable despite aggressive medical management. Peripheral ECMO cannulation initially stabilized his condition, but then his hemodynamics deteriorated with insufficient flow to meet his metabolic needs. Central ECMO along with drainage of his pleural effusions and intraabdominal ascites significantly improved his blood flow from 67 to 88 to 150 to $200 \mathrm{~mL} / \mathrm{kg} / \mathrm{min}$.

After approximately 2 months on ECMO, he was transitioned to $\mathrm{ECCO}_{2} \mathrm{R}$ due to refractory respiratory acidosis. He was completely decannulated approximately 2 weeks later with good neurological recovery. This experience suggests that consideration should be given for the use of central ECMO in pediatric patients who remain hemodynamically insufficient/ unstable due to sepsis while on maximally optimized peripheral ECMO support. Furthermore, $\mathrm{ECCO}_{2} \mathrm{R}$ is a reasonable supportive option in children who exhibit persistent respiratory acidosis while recovering from ARDS.

Funding

Conflict of Interest

None.

\section{References}

1 O'Loughlin RE, Roberson A, Cieslak PR, et al; Active Bacterial Core Surveillance Team. The epidemiology of invasive group A strepto- coccal infection and potential vaccine implications: United States, 2000-2004. Clin Infect Dis 2007;45(07):853-862

2 Nizet V, Arnold JC. Streptococcus pyogenes (group A streptococcus). In: Long SS, Pickering LK, Prober CG, eds. Principles and Practice of Pediatric Infectious Diseases, 4th ed. Chap. 118. China: Elsevier Saunders; 2012:698-707

3 Sande L, Flores AR. Group A, group C, group G beta hemolytic streptococcal infections. In: Cherry J, Demmler-Harrison GJ, Kaplan SL, Steinbach WJ, Hotez P, eds. Feigin and Cherry's Textbook of Pediatric Infectious Diseases, 7th ed. Chap. 82. Philadelphia, PA: Elsevier Saunders; 2014:1140-1152

4 Lithgow A, Duke T, Steer A, Smeesters PR. Severe group A streptococcal infections in a paediatric intensive care unit. J Paediatr Child Health 2014;50(09):687-692

5 American Academy of Pediatrics. Group A streptococcal infections. In: Kimberlin DW, Brady MT, Jackson MA, Long SS, eds. Red Book ${ }^{\circledR}$ : 2015 Report of the Committee on Infectious Diseases. American Academy of Pediatrics; 2015:732-744

6 Low DE. Toxic shock syndrome: major advances in pathogenesis, but not treatment. Crit Care Clin 2013;29(03):651-675

7 Brierley J, Carcillo JA, Choong K, et al. Clinical practice parameters for hemodynamic support of pediatric and neonatal septic shock: 2007 update from the American College of Critical Care Medicine. Crit Care Med 2009;37(02):666-688

8 Conrad SA, Dalton HJ. Extracorporeal life support. In: Nichols DG, ed. Rogers Textbook of Pediatric Intensive Care, 4th ed. Chap. 36. Philadelphia: Lippincott Williams and Wilkins; 2008:544-563

9 Bartlett RH. Extracorporeal support for septic shock. Pediatr Crit Care Med 2007;8(05):498-499

10 MacLaren G, Butt W, Best D. Pediatric septic shock guidelines and extracorporeal membrane oxygenation management. Crit Care Med 2009;37(06):2143-2144, author reply 2144-2145

11 Maclaren G, Butt W, Best D, Donath S, Taylor A. Extracorporeal membrane oxygenation for refractory septic shock in children: one institution's experience. Pediatr Crit Care Med 2007;8(05):447-451

12 Goldman AP, Kerr SJ, Butt W, et al. Extracorporeal support for intractable cardiorespiratory failure due to meningococcal disease. Lancet 1997;349(9050):466-469

13 Pořízka M, Kopecký P, Prskavec T, Kunstýř J, Rulíšek J, Balík M. Successful use of extra-corporeal membrane oxygenation in a patient with streptococcal sepsis: a case report and review of literature. Prague Med Rep 2015;116(01):57-63

14 MacLaren G, Butt W, Best D, Donath S. Central extracorporeal membrane oxygenation for refractory pediatric septic shock. Pediatr Crit Care Med 2011;12(02):133-136

15 MacLaren G, Cove M, Kofidis T. Central extracorporeal membrane oxygenation for septic shock in an adult with H1N1 influenza. Ann Thorac Surg 2010;90(03):e34-e35

16 Richard C, Argaud L, Blet A, et al. Extracorporeal life support for patients with acute respiratory distress syndrome: report of a Consensus Conference. Ann Intensive Care 2014;4:15

17 Ryan DP, Doody DP. Treatment of acute pulmonary failure with extracorporeal support: $100 \%$ survival in a pediatric population. J Pediatr Surg 1992;27(08):1111-1116, discussion 1116-1117

18 Khemani RG, Smith LS, Zimmerman JJ, Erickson S; Pediatric Acute Lung Injury Consensus Conference Group. Pediatric acute respiratory distress syndrome: definition, incidence, and epidemiology: proceedings from the Pediatric Acute Lung Injury Consensus Conference. Pediatr Crit Care Med 2015;16(05, Suppl 1):S23S40

19 Ferguson ND, Fan E, Camporota L, et al. The Berlin definition of ARDS: an expanded rationale, justification, and supplementary material. Intensive Care Med 2012;38(10):1573-1582

20 Ranieri VM, Rubenfeld GD, Thompson BT, et al; ARDS Definition Task Force. Acute respiratory distress syndrome: the Berlin Definition. JAMA 2012;307(23):2526-2533 